\title{
Research on Fractal Characterization of Data Transmission Performance of Hard Disk
}

\author{
Wei Jiang ${ }^{1, *}$, Cuicui $\mathrm{Ji}^{2}$, and Yuntian Dai ${ }^{1}$ \\ ${ }^{1}$ School of Mechanical and Automobile Engineering, Changzhou Institute of Technology, Changzhou, Jiangsu 213032, PR China \\ ${ }^{2}$ School of Mechanical and Electrical Engineering, Hohai University, Changzhou, Jiangsu 213022, PR China
}

\begin{abstract}
The data transfer rate is the traditional criterion for evaluating the overall performance of a hard disk. In this paper, the data transmission performance of hard disk was characterized based on fractal theory. The traditional mechanical hard disks and solid state disks with different capacities and specifications were tested and the corresponding signal graph of data transfer was analysed for the calculation of fractal dimension $D$. The results indicate that the process of hard disk data transfer has fractal characteristics; the fractal dimension $D$ increases with the increase of the average transfer rate and the rotating speed of mechanical hard disk. However, there seems no direct correspondence between the fractal dimension $D$ and the capacity of the hard disk; the larger the floating range of data transmission, the greater value of fractal dimension $D$.
\end{abstract}

\section{Introduction}

"The Fractal Geometry of Nature" was published by French mathematician Mandelbrot in 1982 [1], which played a significant role in promoting fractal geometry and created a new branch of Mathematics - fractal geometry. The objective things have their own hierarchical structure, which means the statistical similarity between the local and the whole in form, function, information, time and space, named as selfsimilarity. Currently, fractal is one of the most influential concepts in the frontier of science and the fractal geometry is an important tool to explore the complexity of structure. Fractal theory is being widely used in various fields as a mathematical tool and many scholars have applied fractal theory to the research of hard disk such as surface topography characterization and simulation, tribological properties of magnetic head, contact deformation between disk and magnetic head and so on [2-4]. Fractal geometry with the nature of irregularity is more appropriate to describe many natural or man-made phenomena than the traditional rule of Euclidean geometry. Furthermore, the fractal theory has been applied to various signal processing and analysis such as vibration signals, heart sound signals and noise signals [5-8]. Fractal theory is an effective tool for describing irregular geometric forms in nonlinear systems. The data transmission of hard disk is a nonlinear process with unstable state of transmission characteristic. In this study, the fractal theory was used for the analysis of data transmission signals of hard disk and the fractal characterization of data transmission performance by calculating the fractal dimension $D$.

\section{Material and method}

The data transfer rate of hard disk reflects the speed of data transmission when hard disk is working and it is the concrete manifestation of the performance of hard disk, which is not static but changing with the specific working circumstances. In reading the data of different tracks and sectors of the hard disk, whether the data is stored continuously or not will affect the data transfer rate of the hard disk. In this study, THINKPAD X220 (Intel Core i5-2520M CPU@2.50 GHz, 8 GB DDR3L $1333 \mathrm{MHz}$, Windows $10 \mathrm{x} 64)$ was used as a testing platform and the HDTunePro (Version 5.5) as a testing tool. Six hard disks with different capacity and specifications were carried out data transmission performance test to obtain the corresponding transmit signals. The hard disks tested including traditional mechanical hard disk and the latest solid state hard disk. The interface of mechanical hard disk is SATA3 and there are two kinds of interface in solid state disk including mSATA and SATA3. Figure 1 demonstrates the data transfer signals of Hitachi $250 \mathrm{~GB}(5400 \mathrm{rpm})$, Hitachi 320 GB (7200 rpm), West Digital (WD) $1 \mathrm{~T}$ (5400 rpm), Lenovo SSD $128 \mathrm{~GB}$, Lenovo SSD $240 \mathrm{~GB}$ and Samsung SSD 512 GB respectively. For the convenience of calculating the fractal dimension $D$, all the data transmission signal curves are normalized.

\footnotetext{
*Corresponding author: jwei@czust.edu.cn
} 

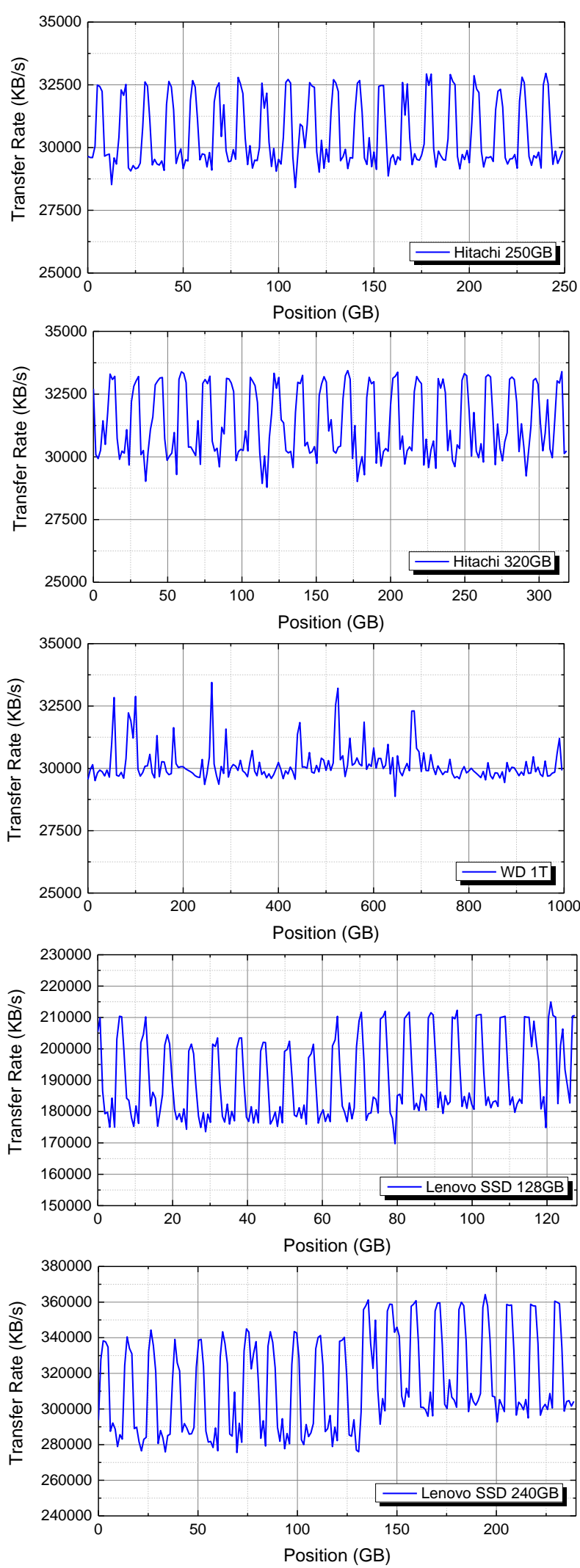

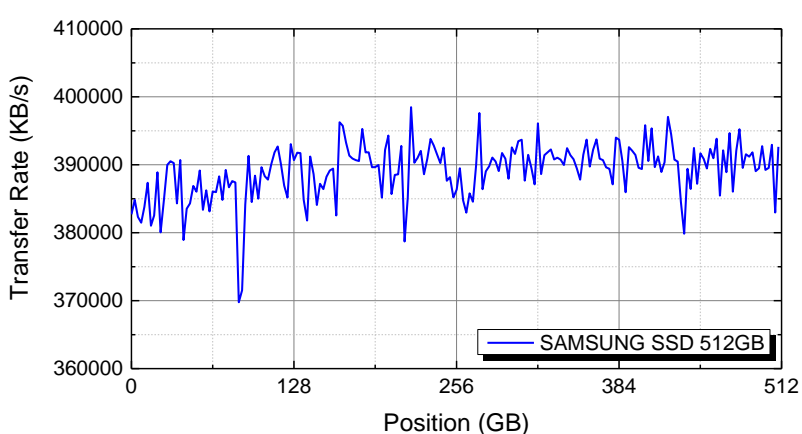

Fig. 1. Data transmission signal curves of different hard disks.

\section{Fractal dimension $D$}

In this study, the fractal dimension $D$ of the data transfer signals of hard disks were calculated using the pixel covering method $[9,10]$. The principle is as follows: transforming the original signal image into grey level image, the fractal feature extraction is mainly based on its grey-scale distribution. By setting the proper grey threshold, a two grey-scale image can be obtained using image binarization processing, thus each pixel in the image will only appear two colours, white or black. Then the boundary is extracted from the feature part, and then the two-valued image is transformed into data file, each of which corresponds to the corresponding pixel location. The value 1 and 0 represent white and black respectively.

Afterwards, the digital data was divided into several parts and the number each rows and columns is $\mathrm{k}$, all the blocks containing value 1 marked as $N(k)$. Assume $\delta^{*}$ as a pixel size, the length of the block would be $\delta=k \delta^{*}$. Least square method was used to carry out linear fitting in double logarithmic coordinates of data points $(\log (N(k)), \log (1 / \delta))$ to calculate the fractal dimension $D$. Based on the principle above, image binarization processing and fractal dimension $D$ calculation was carried out by programming in MATLAB (Version 8.3, Mathworks Inc, USA).

By taking log on both sides, a function is obtained as in Eq. 1:

$$
\log (N(k))=\log (C)+D \log (1 / \delta)
$$

The Fractal dimension $D$ is gained by taking the limit value $\delta \rightarrow 0$ as shown in Eq.2:

$$
D=\lim _{\varepsilon \rightarrow 0} \frac{\log (N(k))}{\log (1 / \delta)}
$$

Figure 2 demonstrates the process of image binarization, boundary extraction and calculation of fractal dimension $D$ of the data transmission signal curve of Hitachi 250 GB hard disk. 


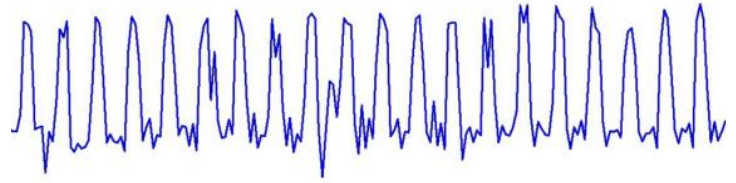

(a) original signal graph

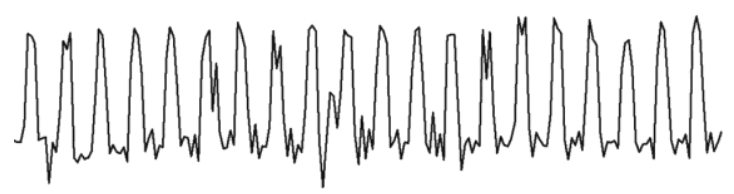

(b) image binarization

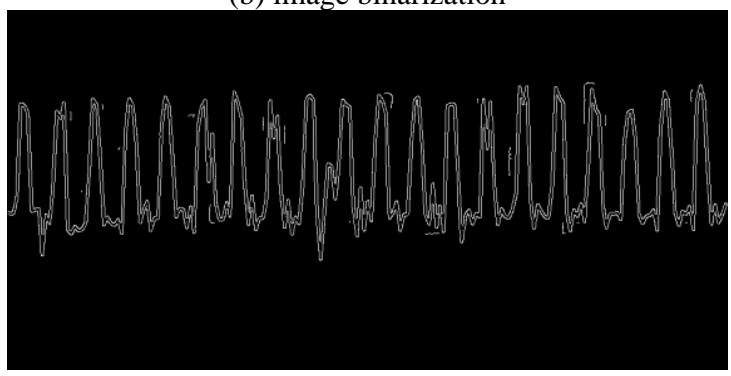

(c) boundary extraction

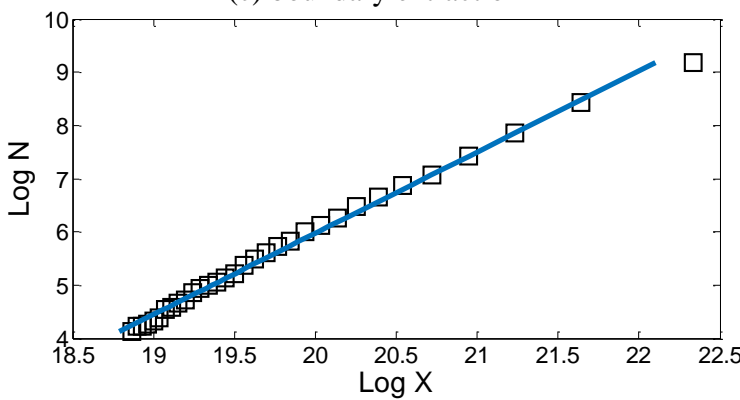

(d) fractal dimension $D$

Fig. 2. Calculation of fractal dimension $D$ of data transfer signal curve.

\section{Results and discussion}

The data transfer rate of a hard disk is divided into external transfer rate and internal transfer rate and there is a buffer between them to alleviate the speed gap. The external data transfer rate refers to the maximum rate that the computer reads data from the cache inside the hard disk. The internal transfer rate can clearly show the read and write speed of hard disk, which is the decisive factor and is the real standard to evaluate the overall performance of a hard disk. The improvement of internal transfer rate is the most direct and most obvious improve to the performance of the disk subsystem. Thus, the hard disk manufacturers are striving to increase the internal transfer rate of hard disks though various techniques such as improving signal processing technology, increasing rotational speed and the most important thing is to continuously improve the capacity of single disk to improve linear density. Because the higher the linear density of the hard disk is, the smaller the seek frequency and the moving distance of the magnetic head, which reduces the average seek time and the internal transfer rate then improved. When the capacity of the single disk is the same, the internal transfer rate increases with the increasing of rotating speed of the hard disk.

Table 1. The performance parameters of the hard disk transmission and the corresponding fractal dimension $D$.

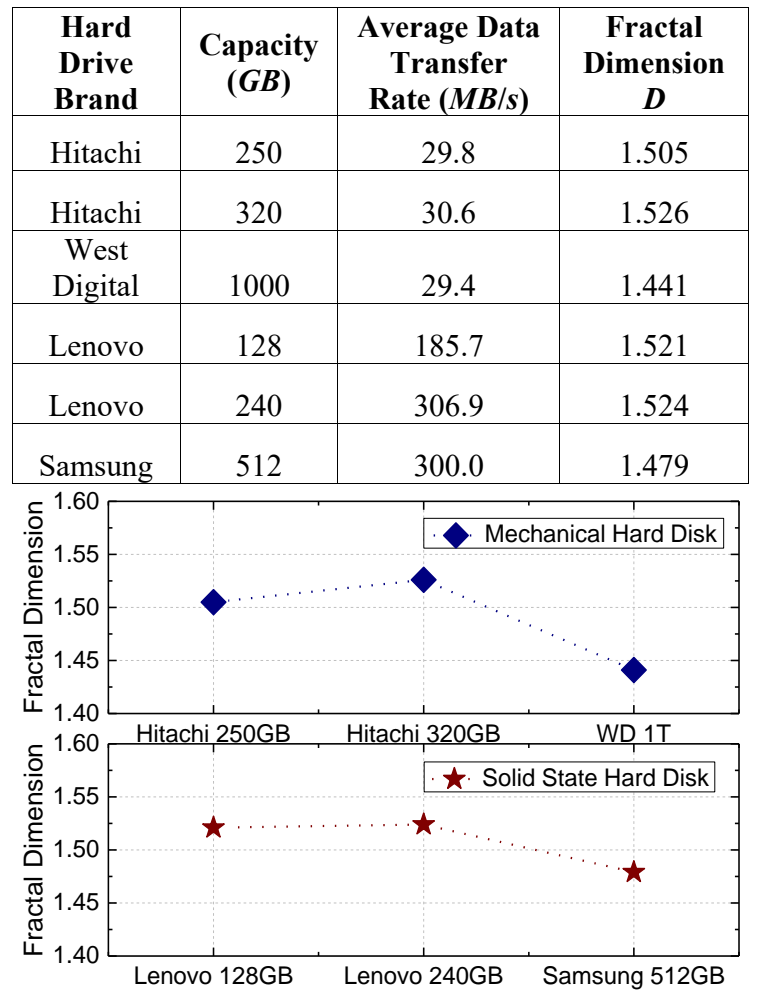

Fig. 3. The relationship between fractal dimension $D$ and the capacity and properties of hard disks.

Table 1 demonstrates the performance parameters of the hard disk transmission and the corresponding fractal dimension $D$, in which the average transfer rate is the average after multiple tests. As illustrated in Table 1 and Figure 3, for traditional mechanical hard disk, the average transfer rate is similar due to the limitation of the interface of the test system. However, with the increasing of rotating speed, the average transfer rate and the corresponding fractal dimension $D$ increased. For solid state hard disk, although there are two different forms of interface such as mSATA and SATA3, the relationship between fractal dimension $D$ and capacity and average transfer rate is not obvious. It is worth noting that the data transmission signal curves of both WD $1 \mathrm{~T}$ and Samsung 512 GB were relatively smooth compared to others, which result in lower fractal dimension $D$. This demonstrates the intrinsic characteristics of fractal, that is fractal dimension $D$ can reflect the complexity of the research object. In addition, despite that the traditional mechanical hard disk and solid state hard disk have great difference in average transfer rate, the value of fractal dimension $D$ is still in a range and did not show significant changes. As a result, 
the mechanical hard disk and solid state disk should be separated for the fractal characterization of the transmission performance.

\section{Conclusion}

Based on the presented analysis and discussion, the following main conclusions can be drawn from this study.

1). The data transmission of hard disk is a nonlinear process, and the data transfer signal curve has fractal characteristics.

$2)$. There is no obvious correspondence between fractal dimension $D$ and the capacity of the hard disk, but the value of fractal dimension $D$ increases with the increasing of the average data transfer rate.

3 ). For mechanical hard disk, the higher the rotating speed, the larger the value of fractal dimension $D$.

4). Whether it is a traditional mechanical hard disk or a solid state hard disk, the greater the fluctuation of the data transmission signal, the greater the fractal dimension $D$.

\section{Conflict of interest statement}

The authors declare that there is no conflict of interest regarding the publication of this paper.

\section{Acknowledgements}

This work was financially supported by the Applied Basic Research Programs of Science and Technology Commission Foundation of Jiangsu Province (BK20150256), the National Natural Science Foundation of China (51505126) and Scientific Research Foundation of Changzhou Institute of Technology (YN1512, YN1626).

\section{References}

1. B. B. Mandelbrot, The Fractal geometry of nature (W. H Freeman and Co, 1982)

2. F. E. Kennedy, C. A. Brown, J. Kolodny, B. M. Sheldon, Fractal analysis of hard disk surface roughness and correlation with static and low-speed friction, J TRIBOL, 121, 4 (1999)

3. T. R. Thomas, B. G. Rosén, Determination of the optimum sampling interval for rough contact mechanics, TRIBOL INT, 33, 9 (2000)

4. H. Ying, M. Zhang, J. C. Liu, Fractal-based automatic localization and segmentation of optic disc in retinal images, IEEE EMBS (2007)

5. A. Sasikanth, S. Wang, Fractal-regular surface characterization based on experimental data from atomic force microscopy of hard disks, ASME IJTC (2007)

6. C. C. Ji, H. Zhu, W. Jiang, A novel method to identify the scaling region for chaotic time series correlation dimension calculation, CHINESE SCI BULL, 56, 9 (2011)

7. C. C. Ji, H. Zhu, W. Jiang, Influence of sampling length and sampling interval on calculating the fractal dimension of chaotic attractors, INT J BIFURCAT CHAOS, 22, 6 (2012)

8. J. R. Wootton, Fractal hard drives for quantum information, NEW J PHYS, 18, 2 (2016)

9. R. D. Peng, H. P. Xie, Y. Ju, Computation method of fractal dimension for 2-D digital image, J CHINA U MIN TECHNO, 33, 1 (2004)

10. W. Jiang, C. C. Ji, H. Zhu, Fractal study on plant classification and identification, IWCFTA (2009) 